Pediatr Radiol. 2015 August ; 45(9): 1323-1332. doi:10.1007/s00247-015-3314-z.

\title{
Analysis of carbonated thin liquids in pediatric neurogenic dysphagia
}

\author{
Jennifer P. Lundine, \\ Department of Speech Pathology, Nationwide Children's Hospital, 700 Children's Drive, \\ Columbus, $\mathrm{OH} 43205$, USA \\ David G. Bates, and \\ Children's Radiological Institute, Nationwide Children's Hospital, Columbus, OH, USA; School of \\ Medicine \& Public Health, Ohio State University, Columbus, OH, USA \\ Han Yin \\ Biostatistics Core, The Research Institute at Nationwide Children's Hospital, Columbus OH, USA
}

\section{Abstract}

Background-Aspiration of liquids is a serious complication of neurological impairments such as traumatic brain injury or stroke. Carbonated liquids have been examined as a possible alternative to thickened liquids to help reduce aspiration in cases of dysphagia in adults, but no published literature to the best of our knowledge has evaluated this technique in children. If carbonated liquids result in safer swallowing in children, they could provide a preferred alternative to thickened liquids.

Objective-This pilot study examined whether carbonated thin liquids (CARB) improved swallowing compared to noncarbonated thin liquids (NOCARB) for children with neurogenic dysphagia.

Materials and methods-Twenty-four children admitted to a level I trauma center for acute neurological injury/disease were evaluated via videofluoroscopic swallow studies. Four descriptive outcome measures were contrasted.

Results-CARB significantly decreased pooling $(P=0.0006)$, laryngeal penetration/aspiration $(P=0.0044)$ and Penetration-Aspiration Scale scores $(P=0.0127)$ when compared to NOCARB. On average, CARB improved scores on the Penetration-Aspiration Scale by 3.7 points for participants who aspirated NOCARB. There was no significant difference in pharyngeal residue noted between CARB and NOCARB $(P=0.0625)$.

Conclusion-These findings support the hypothesis that carbonated thin liquids may provide an alternative to thickened liquids for children with neurogenic dysphagia. Implications for future research and clinical practice are discussed.

J. P. Lundine jennifer.lundine@ nationwidechildrens.org.

Electronic supplementary material The online version of this article (doi:10.1007/s00247-015-3314-z) contains supplementary material, which is available to authorized users.

Conflicts of interest None 


\section{Keywords}

Children; Neurogenic dysphagia; Carbonated liquids; Swallowing; Oropharyngeal dysphagia; Fluoroscopy

\section{Introduction}

Children with neurogenic dysphagia present a unique diagnostic challenge for clinicians, yet published research in this area is lacking [1,2]. The ability to maintain adequate and safe oral nutrition and hydration requires the integration of behavior, cognition and a functional oropharyngeal swallowing mechanism in a highly coordinated, complex act [3]. Acute neurological events, such as traumatic brain injury (TBI) or stroke, can impair swallowing function and place individuals at risk for aspiration and resultant pneumonia [4-7]. Children with neurological impairment have been found to have a high rate of oropharyngeal dysphagia [5], with frequent silent aspiration of thin liquids [6], increasing their likelihood of developing aspiration pneumonia [7]. Because thin liquids are the most easily aspirated, this finding is of primary importance for individuals with an impaired swallow [8].

Radiologic evaluation with videofluoroscopic swallow studies has been proven to be an effective method of imaging the oral, pharyngeal and esophageal phases of the swallow, and it is commonly used with both adults and children when there are concerns for neurogenic dysphagia [4, 9-13].

Findings from previous studies utilizing carbonated thin liquids (CARB) versus noncarbonated thin liquids (NOCARB) in adults show interesting promise in offering a potential alternative to thickened liquids for those individuals with neurogenic dysphagia who aspirate thin liquids. Yet, past research analyzing CARB versus NOCARB in regard to impairments of swallowing has had varying results. More research is needed in this area for both adult and pediatric populations. This study sought to examine whether carbonated liquids might have a positive effect on the swallow of children with neurogenic dysphagia.

This was a phase 1 study of treatment effect [14]. Approval from the Institutional Review Board of Nationwide Children's Hospital was obtained at the outset. There were four research questions considered in the present study of children with neurogenic dysphagia:

1) Will pooling decrease when drinking CARB as compared to NOCARB?

2) Will laryngeal penetration/aspiration decrease when drinking CARB as compared to NOCARB?

3) Will scores on the Penetration-Aspiration Scale [15] decrease when drinking CARB as compared to NOCARB?

4) Will observed pharyngeal residue decrease when drinking CARB as compared to NOCARB? 


\section{Materials and methods}

\section{Participants}

The principal investigator invited participants during a 20-month period. In all cases, the attending physician for the case had referred the participants for a videofluoroscopic swallow study. Prior to the videofluoroscopic swallow study, the principal investigator or an associate involved with the study identified potential participants, informed parents of the study and asked whether they would like to be involved. Inclusion criteria included:

1) Children between the ages of 5 and 18 years of age.

2) Neurological diagnosis necessitating current admission.

3) Indications of a swallowing difficulty for which a videofluoroscopic swallow study was appropriate via findings indicating possible oropharyngeal dysphagia on clinical bedside examination.

4) Ability to tolerate and cooperate with a videofluoroscopic swallow study in general and the investigation's protocol specifically.

Exclusion criteria included any past history of swallowing difficulty prior to the recent neurological diagnoses.

Parents signed consent forms for 30 children recruited for this study. Ultimately, four children who were unable to participate in the videofluoroscopic swallow study for various reasons (e.g., emesis, refusal, gagging on barium) were excluded. Two videofluoroscopic swallow study examinations had technical difficulty during recording that prevented posttesting reviews of the examination results. Twenty-four participants completed this study; their mean age was 13.67 years (range: 5-18; median: 15 ) and all were diagnosed with acute neurological impairments causing neurogenic dysphagia (Table 1).

\section{Methods}

High-density barium sulfate powder (Varibar thin liquid barium sulfate for suspension $40 \%$ w/v after reconstitution; EZ EM Canada Inc., Lake Success, NY) mixed with tap water was the solution used for all stimulus samples. The test materials were prepared in the fluoroscopy suite immediately prior to each study. All materials were given at room temperature (about $22^{\circ} \mathrm{C}$ ). To improve palatability with the pediatric population, $1 \mathrm{Tbsp}$. Nestle Quick (Nestle USA, Inc., Glendale, CA); either chocolate or strawberry, per participant preference) was also added to $100 \mathrm{ml}$ of barium for each consistency tested, as per standard departmental videofluoroscopic swallow study protocol. Lafayette Sparkles effervescent granules (Mallinckrodt Pharmaceuticals, St Louis, MO; sodium bicarbonate, $2.0 \mathrm{~g}$; citric acid, $1.5 \mathrm{~g}$; simethicone and excipients) were added to carbonate the contrast. The granules produce approximately $350 \mathrm{ml}$ of carbon dioxide when added to a liquid. One $4 \mathrm{~g}$ packet of Lafayette Sparkles was added to $100 \mathrm{ml}$ of thin liquid barium contrast plus 1 Tbsp. Nestle Quick suspension. With the exception of the flavoring, this was the same ratio utilized in both the adult studies by Bulow et al. [16] and Sdravou et al. [17]. 
The examination was performed with the use of a GE Legacy Digital Fluoroscopy Unit (GE, Waukesha, WI). The fluoroscopic images were recorded on a 3/4-inch VHS videotape that allowed for real-time, slow-motion and frame-by-frame playback. All participants were evaluated in a seated, upright position and were visualized in the lateral view, to allow for optimal visualization of penetration and aspiration $[10,16]$. The ALARA principle was employed as standard practice. All videos were recorded at 15 frames per s (fps).

One of 11 pediatric radiologists (JR, JO, GB, MH, LL, LB, BA, BC, AF, EH, DD all with post fellowship experience ranging from 5 to 10 years) and a speech-language pathologist (J.L.) with 11 years of experience performed all videofluoroscopic swallow studies. If the first author was not able to attend, an associate investigator with 13 years of experience trained by the first author was in attendance for the study. Prior to beginning the study, participants were asked to swallow each bolus immediately and completely. One of 5 occupational therapists (with experience ranging from 3 to 10 years), trained by the first author regarding the specific methods utilized in this study, administered the test materials to the participants. To indicate which consistency was being given, a lead marker was flashed on the screen indicating the consistency or condition (CARB vs. NOCARB) change prior to the first swallow. While seated, the participant was first given 5 drinks of a $5 \mathrm{ml}$ NOCARB barium bolus by spoon to allow for controlled volume as measured by a graduated syringe. The participants were then administered $60 \mathrm{ml}$ of NOCARB by straw to evaluate their ability to tolerate thin liquids in a more functional presentation. These swallows were observed and rated by the speech-language pathologist to determine diet recommendations but were not measured for purposes of this study. During the evaluation, if aspiration was observed, the presentations of that consistency were ended, putting the participant at no additional risk. This same procedure $(5 \times 5 \mathrm{ml}$ controlled drinks via spoon measured for study purposes and then $60 \mathrm{ml}$ by straw) was repeated with CARB. Barium was carbonated immediately before presentation. If aspiration was noted with NOCARB, participants were assessed using nectar and honey-thick barium as per the standard institutional protocol to help determine appropriate diet recommendations, but these swallows were not measured for purposes of this study. The 24 participants completed the entire protocol.

Descriptive comparisons were made between NOCARB and CARB for the $5 \mathrm{ml}$ swallows only. The following outcome measures were recorded: pooling, penetration/aspiration, scores on the Penetration/Aspiration Scale [15] and pharyngeal residue (Table 2). For purposes of this study, swallow initiation was noted as the point at which laryngeal elevation and opening of the cricopharyngeus were first noted during imaging. Pooling was defined as bolus presence in the valleculae or pyriform sinuses prior to swallow initiation. It was scored relative to its location in the pharyngeal vestibule, and was only scored once contrast was noted prior to swallow initiation in $>1$ swallow to account for some normal variation in swallow timing [18]. Penetration/aspiration were recorded as absent, laryngeal penetration only or aspiration. Penetration was defined as entrance of the bolus into the airway above the level of the true vocal cords, and aspiration was defined as entrance of the bolus into the airway below the level of the true vocal cords. Scores on the Penetration-Aspiration Scale were documented according to standards set by its authors [15]. The reporting of penetration/aspiration was used in addition to reporting of scores on the Penetration- 
Aspiration Scale to allow better comparison to previous studies. Neither Bulow et al. [16] or Krival [19] used the Penetration-Aspiration Scale as an outcome measure, but they did report on the presence or absence of penetration and aspiration. The study by Sdravou et al. [17] is the only published study that has utilized the Penetration-Aspiration Scale when evaluating the effect of CARB on swallowing. While it would seem to be a more reliable outcome measure, the authors wanted to be able to compare results of this study to prior studies that did not use the Penetration-Aspiration Scale. Residue was defined as retention of contrast material in the pharyngeal vestibule following bolus entrance into the esophagus. It was scored based on a protocol established by Eisenhuber et al. [20] that graded residue severity in relation to the perceived height of the retained contrast as referenced against the space available in the valleculae or pyriform sinuses. "Mild" residue was scored as a 1 and indicated that the level of contrast remaining in either the valleculae or pyriform sinuses was less than $25 \%$ of the height of the structure. "Moderate" residue was scored as a 2 and indicated remaining contrast that constituted approximately $25-50 \%$ of the structure's height. "Severe" residue was scored as a 3 and indicated residual contrast that exceeded 50\% of the structure's height.

Once all studies were recorded, the principal investigator reviewed the accumulated 24 exams to ensure that all participants were rated on all measures. Videofluoroscopic swallow study recordings were transferred from VHS to a digital DVD. Time markings were taken from the initial video compilation for each CARB and NOCARB sequence tested for each participant. Each trial analyzed for this study (CARB and NOCARB by spoon) was then given a randomized number and evaluated in this new randomized order. This editing allowed the rater to be blinded to the consistency given for each video observed. A scoring sheet was created that included the trial number with no identifying information regarding participant or condition (CARB vs. NOCARB). Videos were analyzed at normal speed and in slow motion as many times as needed for confident judgment by the first author.. The five swallows were analyzed and recorded for both NOCARB and CARB, but the "worst" (e.g., highest number) was noted for scoring purposes for each outcome variable for each participant.

The data analysis was done using both SPSS (Version 19, Chicago, IL) and SAS (Version 9.3, Cary, NC). Since this study was designed to replicate the prior adult studies of Bulow et al. [16] and Sdravou et al. [17], the Wilcoxon signed rank test was used to test the possible differences between the descriptive outcome measures (pooling, penetration/aspiration, Penetration-Aspiration Scale, residue). The Wilcoxon signed rank test evaluates the difference between two treatments when each participant in the sample is being measured twice [21]. It is possible for a participant to score exactly the same on the two opposing measures (e.g., CARB and NOCARB), and in these cases their score is marked as a "tie" and not utilized in the final analysis. In these cases, the scores were discarded from the analysis thus reducing the sample size [21]. Because of small sample size, $P$-value was calculated based on the exact distribution. The inter-rater and intra-rater reliability were determined by Cohen's kappa coefficient. $P$-values were calculated based on normal approximation or exact distribution as appropriate. An alpha of 0.05 was taken to indicate a statistically significant difference with confidence intervals of $95 \%$ in all tests. 


\section{Results}

\section{Measurement of reliability}

Cohen's $\kappa$ was used to determine the agreement between first and second viewings (as viewed in random order described above) of all 24 participants by the first author on each of the four outcome variables. There was very good agreement between the two ratings for pooling ( $k=0.935, P<0.0005$ ), very good agreement between the two ratings for penetration/ aspiration ( $k=0.882, P<0.0005)$, very good agreement between the two ratings for Penetration-Aspiration Scale $(k=0.924, P<0.0005)$, and very good agreement between the two ratings for residue $(\kappa=0.834, P<0.0005)$.

To estimate inter-rater reliability, one speech-language pathologist with 6 years of experience, who was not involved in this research project, reviewed 33\% $(n=8)$ of the videofluoroscopic swallow studies. This reviewer was not blinded to the intent of the study, but due to video editing methods described above, she was blinded to condition assessed (CARB vs. NOCARB) during reliability assessment. The second reviewer was trained by the first author on the specific outcome measures utilized during this study. Prior to reviewing videos for this study, both the first author and second reviewer independently reviewed videofluoroscopic swallow study exams not used in this study and achieved a $90 \%$ criterion agreement for all outcome measures. The second reviewer has 5 years of experience in videofluoroscopic swallow study analysis and has also been qualified through the institution's training program to perform these examinations. Cohen's $\kappa$ was used to determine the agreement between the two raters' judgments on each of the four outcome variables. There was fair agreement between the two raters for pooling $(k=0.322$, $P=0.2857)$, very good agreement between the two raters for penetration/aspiration ( $k=0.821$, $P<0.0024)$, fair agreement between the two raters for Penetration-Aspiration Scale ( $k=0.355, P=0.0476)$, and moderate agreement between the two raters for residue $(\kappa=0.464$, $P=0.0222)$.

\section{Analysis of outcome variables}

Statistical analysis revealed that pooling of barium within the valleculae and pyriform sinuses prior to the swallow for CARB was noted significantly less often when compared to NOCARB $(P=0.0006)$. Eleven comparisons were noted to have no difference; one participant was noted to have more pooling with CARB than with NOCARB, and the remaining 12 participants were noted to have less pooling with CARB than with NOCARB.

Statistical analysis showed that penetration/aspiration occurred significantly less often with CARB when compared to NOCARB for 10 participants $(P=-0.0044)$. Analysis of penetration/aspiration showed no difference in 13 participants. Twelve participants had no instances of laryngeal penetration with either NOCARB or CARB, and one participant demonstrated aspiration with both liquids. There was increased penetration/aspiration in one patient with CARB compared to NOCARB. Ten participants showed positive effects of CARB compared to NOCARB. An example is shown in the animation clips (Online Resources 1 \& 2). 
Scores on the Penetration-Aspiration Scale were significantly lower (indicating improved swallow function) for swallows with CARB when compared to NOCARB $(P=0.0127)$. Thirteen participants were noted to have no difference on this descriptive measure, and one participant had an increased Penetration-Aspiration Scale score on CARB compared to NOCARB. The remaining 10 participants had improved Penetration-Aspiration Scale scores with CARB compared to NOCARB.

Analysis of residue demonstrated that there is no significant difference in pharyngeal residue between CARB and NOCARB $(P=0.0625)$. With this analysis in particular, it is notable that 19 participants were eliminated for ties. No participants showed greater residue for NOCARB compared to CARB. Five participants retained more residue with CARB than with NOCARB.

As nine of the 10 participants who responded positively to CARB were in the largest diagnostic category (TBI; $n=18$ ), a closer examination of these participants was performed (Table 3). Seven participants were identified to have improved swallows with CARB on the penetration/aspiration variable. Of those seven participants, a percent calculation highlighted the proportion of cases that had scores that improved from the 2-3 range (penetration or aspiration) with NOCARB to a score of 1 (no penetration or aspiration) with CARB. Five of seven participants $(71 \%)$ went from having laryngeal penetration or aspiration (score $=2$ or 3) with NOCARB to no laryngeal penetration or aspiration (score=1) with CARB.

Further examination was completed on participants who showed positive change on the Penetration-Aspiration Scale variable (Table 4). For all participants who had positive change on the Penetration-Aspiration Scale variable $(n=10)$, the average amount of change shown was 3.7 points. In addition, for these same participants, a percent calculation highlighted the proportion of scores that went from the impaired range (score >3) into the normal range (score=1-2.5). Five of ten participants (50\%) went from having Penetration-Aspiration Scale scores that indicated impaired swallows (score>3) with NOCARB into the normal range (score $=1-2.5)$ with CARB.

\section{Discussion}

Currently in clinical practice, practitioners must utilize either compensatory strategies or modifications in diet for individuals with dysphagia [4, 8, 12, 22, 23]. However, in the neurologically impaired patient, there may be cognitive or language deficits that create significant barriers in expected carryover of such compensatory strategies. Furthermore, these strategies may not be developmentally appropriate to use with children. As a result of these barriers, clinicians often must rely on diet modifications to assist in managing swallowing difficulties. These modifications include altering textures or thickening liquids with an artificial thickener to nectar or honey consistency $[8,12,22,23]$. In clinical practice, however, many individuals will protest consuming foods that are altered to different textures. In these cases, patients will often require supplemental tube-feeding as they are not able to meet their caloric or hydration needs by mouth [8, 10, 23]. Thus, if the modified liquids were more acceptable, or alternatives were available, perhaps compliance would improve and medical complications could be reduced. 
One alternative that has been investigated is the addition of carbonation to liquids. In the past two decades, a growing body of literature has demonstrated that swallowing physiology is altered by neuromuscular recruitment when boluses include a sour or chemesthetic property $[16,24-31]$. The mucosa of the pharynx and larynx are covered in receptors that respond to chemesthetic stimuli. Activation of these receptors results in protective reflexes that serve to prevent aspiration [32]. Several studies specifically have examined whether or not carbonation may provide enhanced stimulation to the pharynx to better stimulate the swallow and protect the airway [16, 17, 19, 31,33-36]. The outcome measures evaluated in studies examining the swallows of neurologically impaired adults using carbonated and noncarbonated liquids have included: the incidence of penetration and/or aspiration [16, 17, 31, 35], pharyngeal transit time [16, 31], pharyngeal residue [16], pooling [31, 35], laryngeal elevation [31] and scores on the Penetration-Aspiration Scale [15]. Results have varied, with no consistent findings from study to study. Studies by Swank-Nixon [31], Bulow et al. [16] and Sdravou et al. [17] have all reported positive findings on at least some outcome measures. Only Newman et al. [35] have evaluated the effect of carbonation in children (age range $=2-63$ months). Results from this study found that carbonation decreased the incidence of penetration and pooling but did not decrease the incidence of aspiration. The current study examined the effect of carbonation on pediatric individuals following a neurological event.

Bolus position at swallow initiation is known to be variable in normal eating and swallowing [37], though the swallow should be initiated prior to bolus entrance into the pyriform sinuses for individuals younger than 50 [18]. Between the ages of 5 to 8 years, children typically achieve an adult pattern of muscle activation during the oral and pharyngeal phases of swallowing [38]. Recent studies with adults have revealed that pre-swallow liquid bolus entry into the pharynx (particularly at the level of the valleculae) occurs in healthy individuals $[18,37]$. Pooling in this study was examined as a means to observe the effect of carbonation on swallow initiation. CARB significantly reduced pooling when compared to NOCARB $(P=0.002)$. In fact, in 11 of 12 cases $(92 \%)$ where CARB demonstrated positive effects, pooling changed from abnormal (score of $4=$ vallecular+pyriform) to within normal limits (score of $1=$ none or $2=$ valleculae only). These results suggest faster swallow initiation or pharyngeal transit time, though specific time measurements were not calculated in this study. Results are similar to the work of Bulow et al. [16], whose study showed reduced pharyngeal transit time, and indicated less of a swallow initiation delay when using CARB.

Aspiration that is visible on videofluoroscopic swallow studies is associated with increased risk of aspiration pneumonia or airway obstruction [37, 39]. Weir et al. [7] revealed that children with silent aspiration were 3.7 times more likely to have neurological impairment than those who aspirated with a cough, and that $81 \%$ of children with neurological impairment had silent aspiration. Morgan et al. [5] identified silent aspiration as a significant issue in the rehabilitation of children with acquired brain injuries. Reducing the risk of aspiration for these patients and allowing them access to non-thickened liquids would be a critical breakthrough in dysphagia management. Results of this study found that CARB significantly reduced the instances of penetration/aspiration $(P=0.008)$ when compared to 
NOCARB. These results are similar to previous studies comparing CARB to NOCARB in adults $[16,17,31]$.

Impaired swallowing is indicated by either laryngeal penetration or aspiration, as shown by a score of 2 or 3 on the penetration/aspiration variable. When participants were noted to have impaired scores on the penetration/aspiration variable (see Table $4 ; n=12$ ), all except two participants (patients 2 and 15) showed positive improvement with CARB compared to NOCARB. Ten of 12 participants (83\%) demonstrated improvements on this variable, and seven of 12 participants (58\%) who scored a 2 or 3 on NOCARB trials improved their swallows to a score of 1 , indicating no laryngeal penetration or aspiration with CARB.

The Penetration-Aspiration Scale is a quantifiable way to rate dysphagia beyond simply the presence or absence of penetration or aspiration [15, 40]. While the Penetration-Aspiration Scale has not been applied consistently in pediatric swallowing research [41], it has been shown to be reliable [42] and has been used in prior studies [43, 44] with children. Adults without dysphagia have an average Penetration-Aspiration Scale score of 1.12 (range: 1-2.5). High scores (>4) represent significant dysphagia [45]. In this study, there was a significant effect of CARB on the Penetration-Aspiration Scale compared to NOCARB with the Wilcoxon signed rank test $(P=0.0127)$. For all participants who had change on the Penetration-Aspiration Scale, all except one showed a positive change (see Table 4). For those participants whose swallows improved on the Penetration-Aspiration Scale, their swallows improved by an average of 3.7 points. For $50 \%$ of participants (patients 12, 18, 19, 20 and 29), CARB improved Penetration-Aspiration Scale scores from the impaired range (>3) into the average range (1-2.5). Lastly, two of ten participants (20\%), while showing positive change on the Penetration-Aspiration Scale variable, remained within the severely impaired range with CARB trials (patients 17 and 21). For the participant who did aspirate on CARB (patient 15), there was no notable difference in her presentation that would seem to explain this result.

It was hypothesized that pharyngeal retention would be reduced significantly in response to CARB. However, no significant difference was found between CARB and NOCARB $(P=0.0625)$. Results for this variable may actually be less conclusive due to the high number of ties within the ranked data $(n=19)$, leaving only five participants for the final statistic calculation. One possible explanation for the high number of ties could be that the bolus presentation was not counterbalanced. All participants received NOCARB prior to receiving CARB. If a participant had initial residue with NOCARB, which was not fully cleared before CARB was trialed, this may have led to additional buildup of residue within the pharynx. Had variables been counterbalanced, a different effect may have been observed. Of note, in prior adult studies [16, 17], variables were not counterbalanced.

Notably, three participants (patients 4, 6 and 11) showed very little difference on any variable. Participant 11 demonstrated slight improvement on 3 of 4 variables (pooling, penetration/aspiration and Penetration-Aspiration Scale), but arguably was within normal limits for Penetration-Aspiration Scale with a score of 2. The etiology of each of these participants' neurogenic dysphagia was stroke. It may be that this study's sample from the pediatric stroke population was too small. Distinct etiologies or site of injury may respond 
differently to this chemesthetic stimulus. Krival [19] reported post-stroke adults in her study did not show significant effects of CARB, whereas Bulow et al. [16] included adults poststroke in his study group, and showed positive effects of CARB on variables such as penetration/aspiration. With the small $n$ and the normal swallows of the post-stroke participants in this study, it is difficult to draw any significant conclusions. It would be worthwhile to include additional neurological diagnoses in a larger study of neurogenic dysphagia to investigate further how CARB may impact dysphagia based on etiology or to focus studies on one diagnosis at a time. Perhaps the chemesthetic properties of the stimulus have different effects depending on the etiology of neurogenic dysphagia. This study provides an interesting new perspective not previously investigated in that it has a larger population of diffuse injuries (TBI) than prior studies. In this sample, $90 \%$ of the participants who responded positively to CARB were in the TBI category.

There are some limitations of this study that must be considered when designing further research in this area. Larger population samples are needed to help draw more conclusive results, in particular including more participants who have impaired swallowing as demonstrated on videofluoroscopic swallow studies to strengthen the eventual conclusions, perhaps using a prospective, case-control design. Further, inclusion of more participants at the younger end of the age range would strengthen eventual findings, as the age distribution in this study was skewed toward higher ages. A blinded review process and counterbalancing of the experimental conditions should be considered as well. Counterbalanced presentation may help avoid a bias affecting the various outcome variables, as the order of presentation may have affected more than just the residue outcome. Inclusion of swallows of both NOCARB and CARB by straw during this study, though not specifically measured, allowed speech-language pathologists to evaluate participants for functional drinking to inform diet recommendations but may have contributed to fatigue or other potential confounding variables. Consideration should be taken to evaluate study variables separately, and then utilize standard assessment procedures to establish a safe diet for participants. Enhanced technology would allow for the inclusion of more refined measurements for variables such as pharyngeal transit time (e.g., Bulow et al. [16] and Sdravou et al. [17]) and pharyngeal residue (e.g., Sdravou et al. [17] and Pearson et al. [46]), and may enhance reliability for measurements such as pooling and residue.

The recording rate used in this study was based on an American College of Radiology white paper summary [47] and papers discussing the application of the ALARA principle in pediatric studies (e.g., Strauss \& Kaste [48] and Cohen [49]). Since the completion of data collection for this project, one study has been published that found increased likelihood of not detecting supraglottic penetration of barium using $15 \mathrm{fps}$ in videofluoroscopic swallow studies with children [50]. It is a possible limitation of this study that the recording rate may have resulted in missed instances of laryngeal penetration. No studies have been published that show missed aspiration events in children using $15 \mathrm{fps}$, though it is now a standard recommendation that videofluoroscopic swallow studies using digital equipment record at 30 fps to most clearly identify swallowing impairment and make treatment recommendations in adult studies (e.g., Bonilha et al. [51]). 
Additional directions for future research include larger or varied volumes of barium (e.g., Sdravou et al. [17]) with children and adolescents using CARB to ensure, for example, that children would be safe to drink a carbonated liquid through a straw once they return to their unit on the hospital floor. As most studies, including the present study, have examined the effects of barium after immediate carbonation, it will be important to monitor carbonated beverages to determine how rapidly the carbon dioxide concentration changes within the liquid. Researchers may need to further determine if a less-carbonated beverage affects swallowing performance. Perhaps there is an optimal level of carbonation needed to stimulate the mechanoreceptors, chemoreceptors, nociceptors and thermoreceptors in the pharynx and larynx to stimulate the protective reflexes that aid in aspiration prevention [32].

Even if CARB does prove in the future to be an effective treatment strategy for neurogenic dysphagia, it will need to be carefully assessed on a case-by-case basis using videofluoroscopic swallow studies. As seen in this study, there was one participant (patient 15) who aspirated CARB after safely handling NOCARB. Each individual with dysphagia must be individually evaluated to ensure that CARB will affect his/her swallowing performance positively [17].

For children and adolescents with swallowing impairments, CARB could offer a considerable change to dysphagia management. Use of CARB may reduce use of thickened liquids and could decrease the length of time that children require nasogastric tubes for feeding and/or hydration. Patients with TBI frequently pull out their feeding tubes, necessitating repeated replacement. This is uncomfortable for the child, difficult for the parents and/or guardians to observe and creates additional medical risks for the child, including radiation exposure. If this risk could be reduced for these children, CARB has the potential to change the individual's behavior for the positive and improve quality of life. Additionally, for those children who must leave the hospital using thickened liquids, the cost of thickeners can be a financial burden for their families, as these are not usually covered by insurance [52]. For some families, this leads to non-compliance, simply because they cannot afford to purchase the thickeners their child needs. If children are able to drink carbonated flavored water instead of using thickeners, this could be a significant financial savings for families and avoids putting the child at unnecessary risk for medical complications related to non-compliance.

Results from this pilot study show that CARB appears to have a significant effect on neurogenic dysphagia in the pediatric population, though further research is needed. These findings could be expanded in a larger pediatric study with more participants and subgroup analyses to see specifically how CARB affects the swallow following stroke or brain injury compared to other etiologies, such as infection or tumor resection. While perhaps not appropriate for all individuals (e.g., people with severe gastrointestinal reflux disease), future research should establish if CARB impacts patient hydration, shortens the length of time needed for supplementary tube feeding, and improves quality of life for children and adolescents with neurogenic dysphagia. 


\section{Conclusion}

The results of this study add support to the growing body of literature that carbonation shows promise in reducing penetration and aspiration in neurogenic dysphagia, which may not apply only to adults but may also be true for children and adolescents. This study takes an important step in moving forward an underrepresented area of dysphagia research, by identifying children and adolescents with neurogenic dysphagia who appear to benefit from carbonation when compared to non-carbonated liquids.

\section{Supplementary Material}

Refer to Web version on PubMed Central for supplementary material.

\section{Acknowledgments}

This project was funded in part by the Maternal and Child Health Bureau Grant T73MC00049 and CTSA grant UL1TR001070.

Jennifer P. Lundine would like to thank the following people for their support with the organization and analysis of this study: Kelsey Egelhoff, $\mathrm{PhD}$, Michael Trudeau, PhD, Linda Lowes, PhD, Adriane Baylis, PhD, Tamela Thompson, MA, Stephanie Oswald, MA, and Caitlin Sirois, MS. Much gratitude to Brad Hoehne in the Department of Radiology for his assistance with the video edits required for this study.

\section{References}

1. Morgan AT. Dysphagia in childhood traumatic brain injury: a reflection on the evidence and its implications for practice. Dev Neurorehabil. 2010; 3:192-203. [PubMed: 20450469]

2. Plowman EK, Mehdizadeh O, Leder SB, et al. A bibliometric review of published abstracts presented at the dysphagia research society: 2001-2011. Dysphagia. 2012; 28:128-130.

3. Ylvisaker, M.; Logemann, JA. Therapy for feeding and swallowing disorders following head injury. In: Ylsivaker, M., editor. Head injury rehabilitation: children and adolescents. College-Hill Press; Boston: 1985. p. 195-215.

4. Dodds WJ, Logemann JA, Stewart E. Radiologic assessment of abnormal oral and pharyngeal phases of swallowing. AJR Am J Roentgenol. 1990; 5:965-974. [PubMed: 2108570]

5. Morgan A, Ward E, Murdoch B, et al. Incidence, characteristics, and predictive factors for dysphagia after pediatric traumatic brain injury. J Head Trauma Rehabil. 2003; 3:239-251. [PubMed: 12802166]

6. Arvedson J, Rogers B, Buck G, et al. Silent aspiration prominent in children with dysphagia. Int J Pediatr Otorhinolaryngol. 1994; 2-3:173-181. [PubMed: 8157416]

7. Weir K, McMahon S, Barry L, et al. Oropharyngeal aspiration and pneumonia in children. Pediatr Pulmonol. 2007; 11:1024-1031. [PubMed: 17893917]

8. Dusick A. Investigation and management of dysphagia. Semin Pediatr Neurol. 2003; 4:255-264. [PubMed: 14992457]

9. Carrau, RL.; Murry, T., editors. Comprehensive management of swallowing disorders. Plural Publishing; San Diego: 1999.

10. Arvedson JC. Assessment of pediatric dysphagia and feeding disorders: clinical and instrumental approaches. Dev Disabil Res Rev. 2008; 2:118-127. [PubMed: 18646015]

11. Rogers B, Arvedson J. Assessment of infant oral sensorimotor and swallowing function. Ment Retard Dev Disabil Res Rev. 2005; 1:74-82. [PubMed: 15856438]

12. Tutor JD, Gosa MM. Dysphagia and aspiration in children. Pediatr Pulmonol. 2012; 4:321-337. [PubMed: 22009835] 
13. Smith Hammond CA, Goldstein LB. Cough and aspiration of food and liquids due to oralpharyngeal dysphagia: ACCP evidence-based clinical practice guidelines. Chest. 2006; 129:154S168S. [PubMed: 16428705]

14. Robey RR. A five-phase model for clinical-outcome research. J Commun Disord. 2004; 5:401411. [PubMed: 15231420]

15. Rosenbek JC, Robbins JA, Roecker EB, et al. A penetration-aspiration scale. Dysphagia. 1996; 2:93-98. [PubMed: 8721066]

16. Bulow M, Olsson R, Ekberg O. Videoradiographic analysis of how carbonated thin liquids and thickened liquids affect the physiology of swallowing in subjects with aspiration on thin liquids. Acta Radiol. 2003; 44:366-372. [PubMed: 12846685]

17. Sdravou K, Walshe M, Dagdilelis L. Effects of carbonated liquids on oropharyngeal swallowing measures in people with neurogenic dysphagia. Dysphagia. 2012; 2:240-250. [PubMed: 21822745]

18. Martin-Harris B, Brodsky MB, Michel Y, et al. Delayed initiation of the pharyngeal swallow: normal variability in adult swallows. J Speech Lang Hear Res. 2007; 3:585-594. [PubMed: 17538102]

19. Krival, CR. Dissertation. University of Cincinnati; 2007. Effects of carbonated vs. thin and thickened liquids on swallowing in adults with neurogenic oropharyngeal dysphagia. http:// etd.ohiolink.edu

20. Eisenhuber E, Schima W, Schober E, et al. Videofluoroscopic assessment of patients with dysphagia: pharyngeal retention is a predictive factor for aspiration. AJR Am J Roentgenol. 2002; 178:393-398. [PubMed: 11804901]

21. Gravetter, FJ.; Wallnau, LB. Statistics for the behavioral sciences. Wadsworth Cengage Learning; Belmont: 2009.

22. Arvedson JC. Management of pediatric dysphagia. Otolaryngol Clin N Am. 1998; 3:453-476.

23. Prasse JE, Kikano GE. An overview of pediatric dysphagia. Clin Pediatr (Phila). 2009; 48:247251. [PubMed: 19023104]

24. Ding R, Logemann JA, Larson CR, et al. The effects of taste and consistency on swallow physiology in younger and older healthy individuals: a surface electromyographic study. J Speech Lang Hear Res. 2003; 46:977-989. [PubMed: 12959474]

25. Leow LP, Huckabee ML, Sharma S, et al. The influence of taste on swallowing apnea, oral preparation time, and duration and amplitude of submental muscle contraction. Chem Senses. 2007; 32:119-128. [PubMed: 17071940]

26. Todd JT, Butler SG, Plonk DP. Effects of chemesthetic stimuli mixtures with barium on swallowing apnea duration. Laryngoscope. 2012; 10:2248-2251. [PubMed: 22961333]

27. Logemann JA, Pauloski BR, Colangelo L, et al. Effects of a sour bolus on oropharyngeal swallowing measures in patients with neurogenic dysphagia. J Speech Hear Res. 1995; 38:556563. [PubMed: 7674647]

28. Pelletier CA, Lawless HT. Effect of citric acid and citric acid-sucrose mixtures on swallowing in neurogenic oropharyngeal dysphagia. Dysphagia. 2003; 4:231-241. [PubMed: 14571326]

29. Chee C, Arshad S, Singh S, et al. The influence of chemical gustatory stimuli and oral anaesthesia on healthy human pharyngeal swallowing. Chem Senses. 2005; 5:393-400. [PubMed: 15829608]

30. Pelletier CA, Dhanaraj GE. The effect of taste and palatability on lingual swallowing pressure. Dysphagia. 2006; 2:121-128. [PubMed: 16703444]

31. Swank Nixon, T. Network: a newsletter of dietetics in physical medicine and rehabilitation. American Dietetic Association; New York: 1997. Use of carbonated liquids in the treatment of dysphagia.

32. Bradley RM. Sensory receptors of the larynx. Am J Med. 2000; 108:47S-50S. [PubMed: 10718452]

33. Mastan A, Michou E, Mistry S, et al. Evidence for an enhancing effect of carbonated liquids on complex human swallowing behavior. Gut. 2011; 60:A162.

34. Michou E, Mastan A, Ahmed S, et al. Examining the role of carbonation and temperature on water swallowing performance: a swallowing reaction-time study. Chem Senses. 2012; 37:799-807. [PubMed: 22843761] 
35. Newman L, Armstrong R, Rogers T, et al. The effect of carbonation on sensory dysphagia in the pediatric population: abstracts of scientific papers presented at the Ninth Annual Dysphagia Research Society Meeting, Savannah, Georgia, USA, October 26-28, 2000. Dysphagia. 2001; 16:146-150.

36. Krival K, Bates C. Effects of club soda and ginger brew on linguapalatal pressures in healthy swallowing. Dysphagia. 2012; 2:228-239. [PubMed: 21811834]

37. Matsuo K, Palmer JB. Anatomy and physiology of feeding and swallowing: normal and abnormal. Phys Med Rehabil Clin N Am. 2008; 4:691-707. vii. [PubMed: 18940636]

38. Stephen JR, Taves DH, Smith RC, et al. Bolus location at the initiation of the pharyngeal stage of swallowing in healthy older adults. Dysphagia. 2005; 4:266-272. [PubMed: 16633870]

39. Marik PE. Aspiration pneumonitis and aspiration pneumonia. N Engl J Med. 2001; 9:665-671. [PubMed: 11228282]

40. Hind JA, Gensler G, Brandt DK, et al. Comparison of trained clinician ratings with expert ratings of aspiration on videofluoroscopic images from a randomized clinical trial. Dysphagia. 2009; 2:211-217. [PubMed: 18953607]

41. Rosenbek, JC.; Power, M.; Lefton-Greif, M., et al. Translation to treatment: the clinical implications of penetration/aspiration. Clinical presentation at the Annual Convention of the American Speech Hearing Association; Miami, Florida. 16-18 Nov 2006; 2006.

42. Gosa, MM.; Suiter, DM. Reliability of the penetration-aspiration scale for use with pediatric populations. Paper presented at the Annual Convention of the American Speech Hearing Association; San Diego. 16-19 Nov 2011; 2011.

43. Weir KA, McMahon S, Taylor S, et al. Oropharyngeal aspiration and silent aspiration in children. Chest. 2011; 140:589-597. [PubMed: 21436244]

44. Osborn AJ, de Alarcon A, Tabangin ME, et al. Swallowing function after laryngeal cleft repair: more than just fixing the cleft. Laryngoscope. 2014; 124:1965-1969. [PubMed: 25098751]

45. Daniels SK, Schroeder MF, McClain M, et al. Dysphagia in stroke: development of a standard method to examine swallowing recovery. J Rehabil Res Dev. 2006; 3:347-356. [PubMed: 17041820]

46. Pearson WG, Molfenter SM, Smith ZM, et al. Image-based measurement of post-swallow residue: the normalized residue ratio scale. Dysphagia. 2013; 28:167-177. [PubMed: 23089830]

47. Amis ES, Butler PF, Applegate KE, et al. American College of Radiology white paper on radiation dose in medicine. J Am Coll Radiol. 2007; 4:272-284. [PubMed: 17467608]

48. Strauss KJ, Kaste SC. The ALARA (as low as reasonably achievable) concept in pediatric interventional and fluoroscopic imaging: Striving to keep radiation dose as low as possible during fluoroscopy of pediatric patients - a white paper executive summary. Pediatr Radiol. 2006; 36:110-112. [PubMed: 16862422]

49. Cohen MD. Are we doing enough to minimize fluoroscopic radiation exposure in children? Pediatr Radiol. 2007; 37:1020-1024. [PubMed: 17673995]

50. Cohen MD. Can we use pulsed fluoroscopy to decrease the radiation dose during video fluoroscopic feeding studies in children? Clin Radiol. 2009; 64:70-73. [PubMed: 19070700]

51. Bonilha HS, Blair J, Carnes B, et al. Preliminary investigation of the effect of pulse rate on judgments of swallowing impairment and treatment recommendations. Dysphagia. 2013; 28:528538. [PubMed: 23559454]

52. Ohio Administrative Code. [Accessed 20 Jan 2013] Chap5101:3-10-26: medical supplies, durable medical equipment, orthoses, and prosthesis providers: enteral nutritional products. 2011. http:// codesohiogov/oac/5101\%3A3-10-26, 2011 


\section{Table 1}

Participants' characteristics $(n=24)$

\begin{tabular}{|c|c|c|c|c|}
\hline Diagnosis & $\begin{array}{r}\text { Participant } \\
\text { code }\end{array}$ & $\begin{array}{r}\text { Age } \\
\text { (years) }\end{array}$ & Gender & $\begin{array}{l}\text { Weeks post onset } \\
\text { of diagnosis }\end{array}$ \\
\hline \multirow[t]{18}{*}{ TBI } & 1 & 15 & $\mathrm{M}$ & 1 \\
\hline & 3 & 15 & M & 7 \\
\hline & 7 & 5 & M & 2 \\
\hline & 12 & 15 & M & 9 \\
\hline & 14 & 8 & M & 2 \\
\hline & 16 & 14 & $\mathrm{M}$ & 7 \\
\hline & 17 & 16 & M & 5 \\
\hline & 18 & 17 & $\mathrm{M}$ & 2 \\
\hline & 22 & 13 & M & 3 \\
\hline & 23 & 15 & M & 2 \\
\hline & 24 & 15 & M & 1 \\
\hline & 19 & 18 & $\mathrm{~F}$ & 6 \\
\hline & 2 & 6 & $\mathrm{~F}$ & 2 \\
\hline & 8 & 17 & $\mathrm{~F}$ & 5 \\
\hline & 10 & 6 & $\mathrm{~F}$ & 2 \\
\hline & 15 & 15 & $\mathrm{~F}$ & 3 \\
\hline & 21 & 17 & $\mathrm{~F}$ & 5 \\
\hline & 26 & 17 & $\mathrm{~F}$ & 7 \\
\hline Anoxic brain injury & 29 & 16 & $\mathrm{M}$ & 8 \\
\hline CVA Ischemic & 4 & 17 & $\mathrm{~F}$ & 5 \\
\hline Hemorrhagic/Ischemic & 6 & 12 & $\mathrm{~F}$ & 2 \\
\hline Ischemic & 11 & 13 & $\mathrm{~F}$ & 3 \\
\hline Encephalitis & 9 & 10 & $\mathrm{M}$ & 6 \\
\hline $\begin{array}{l}\text { Posterior fossa tumor } \\
\text { resection }\end{array}$ & 20 & 16 & $\mathrm{~F}$ & 4 \\
\hline
\end{tabular}

TBI Traumatic brain injury, $C V A$ Cerebral vascular accident, $M$ Male, $F$ Female 
Table 2

Definitions of the descriptive outcome measures

\begin{tabular}{|c|c|}
\hline $\begin{array}{l}\text { Descriptive } \\
\text { measure }\end{array}$ & Definition \\
\hline \multirow[t]{4}{*}{ Pooling } & $1=$ None \\
\hline & $\begin{array}{l}2=\text { Bolus pools in valleculae prior to swallow } \\
\text { initiation }\end{array}$ \\
\hline & $\begin{array}{l}3=\text { Bolus pools in pyriform sinuses prior to } \\
\text { swallow initiation }\end{array}$ \\
\hline & $\begin{array}{l}4=\text { Bolus pools in both valleculae and pyriform } \\
\text { prior to swallow initiation }\end{array}$ \\
\hline \multirow[t]{3}{*}{ Penetration/aspiration } & $1=$ No laryngeal penetration or aspiration \\
\hline & 2=Laryngeal penetration \\
\hline & $3=$ Aspiration \\
\hline $\begin{array}{l}\text { Penetration- } \\
\text { Aspiration }\end{array}$ & $1=$ Material does not enter the airway \\
\hline \multirow[t]{7}{*}{ Scale [32] } & $\begin{array}{l}2=\text { Material enters the airway, remains above the } \\
\text { vocal folds and is ejected from the airway }\end{array}$ \\
\hline & $\begin{array}{l}3=\text { Material enters the airway, remains above the } \\
\text { vocal folds and is not ejected from the airway }\end{array}$ \\
\hline & $\begin{array}{l}4=\text { Material enters the airway, contacts the vocal } \\
\text { folds and is ejected from the airway }\end{array}$ \\
\hline & $\begin{array}{l}5=\text { Material enters the airway, contacts the vocal } \\
\text { folds and is not ejected from the airway }\end{array}$ \\
\hline & $\begin{array}{l}6=\text { Material enters the airway, passes below the } \\
\text { vocal folds and is ejected into the larynx or } \\
\text { out of the airway }\end{array}$ \\
\hline & $\begin{array}{l}\text { 7=Material enters the airway, passes below the } \\
\text { vocal folds and is not ejected from the trachea } \\
\text { despite effort }\end{array}$ \\
\hline & $\begin{array}{c}8=\text { Material enters the airway, passes below the } \\
\text { vocal folds and no effort is made to eject }\end{array}$ \\
\hline \multirow[t]{4}{*}{ Pharyngeal residue } & $1=$ No residue \\
\hline & $\begin{array}{l}2=\text { Mild residue (residue is less than } 25 \% \text { of the } \\
\text { height of the space containing it) }\end{array}$ \\
\hline & $\begin{array}{c}3=\text { Moderate residue (residue is between } 25 \% \text { and } \\
50 \% \text { of the height of the space containing it) }\end{array}$ \\
\hline & $\begin{array}{l}4=\text { Severe residue (residue is less greater than } \\
50 \% \text { of the height of the space containing it) }\end{array}$ \\
\hline
\end{tabular}

Pediatr Radiol. Author manuscript; available in PMC 2016 August 01. 
Table 3

Comparison of PENASP for NOCARB and CARB for TBI participants $(n=18)$

\begin{tabular}{llc}
\hline $\begin{array}{l}\text { Participant } \\
\text { code }\end{array}$ & $\begin{array}{l}\text { PENASP response } \\
\text { on NOCARB }\end{array}$ & $\begin{array}{c}\text { PENASP response } \\
\text { on CARB }\end{array}$ \\
\hline 1 & 1 & 1 \\
2 & 3 & 3 \\
3 & 1 & 1 \\
7 & 1 & 1 \\
8 & 1 & 1 \\
10 & 1 & 1 \\
12 & $\mathbf{3}$ & $\mathbf{1}^{a}$ \\
14 & 1 & 1 \\
15 & $\mathbf{2}$ & $\mathbf{3}^{b}$ \\
16 & $\mathbf{2}$ & $\mathbf{1}^{a}$ \\
17 & $\mathbf{3}$ & $\mathbf{2}^{a}$ \\
18 & $\mathbf{2}$ & $\mathbf{1}^{a}$ \\
19 & $\mathbf{3}$ & $\mathbf{1}^{a}$ \\
21 & $\mathbf{3}$ & $\mathbf{2}^{a}$ \\
22 & 1 & $1^{a}$ \\
23 & 1 & $1^{a}$ \\
24 & $\mathbf{2}$ & $\mathbf{1}^{a}$ \\
26 & 1 & \\
\hline
\end{tabular}

$C A R B$ carbonated thin liquids, NOCARB noncarbonated thin liquids, PENASP Penetration-Aspiration Scale, TBI traumatic brain injury See Table 2 for definitions of outcome measures

${ }^{a}$ Indicates patient swallows improved with CARB

$b_{\text {Indicates patient swallows worsened with CARB }}$

Bold text indicates change 


\section{Table 4}

Comparison of NOCARB and CARB for all outcome variables (pooling, penetration/aspiration, PenetrationAspiration Scale, residue) and change on Penetration-Aspiration Scale variable for each participant $(n=24)$

\begin{tabular}{|c|c|c|c|c|c|c|c|c|c|}
\hline \multirow[t]{2}{*}{ Participant code } & \multicolumn{2}{|l|}{ Pooling } & \multicolumn{2}{|c|}{ Penetration/aspiration } & \multicolumn{2}{|c|}{ Penetration-Aspiration Scale } & \multirow[t]{2}{*}{ Change on scale } & \multicolumn{2}{|l|}{ Residue } \\
\hline & NOCARB & CARB & NOCARB & CARB & NOCARB & CARB & & NOCARB & CARB \\
\hline 1 & 4 & 4 & 1 & 1 & 1 & 1 & 0 & 1 & 1 \\
\hline 2 & 2 & 2 & 3 & 3 & 8 & 8 & 0 & 1 & 1 \\
\hline 3 & 2 & $\mathbf{1}^{a}$ & 1 & 1 & 1 & 1 & 0 & 2 & 2 \\
\hline 4 & 1 & 1 & 1 & 1 & 1 & 1 & 0 & 2 & 2 \\
\hline 6 & 1 & 1 & 1 & 1 & 1 & 1 & 0 & 2 & 2 \\
\hline 7 & 4 & $2^{a}$ & 1 & 1 & 1 & 1 & 0 & 1 & $2^{b}$ \\
\hline 8 & 4 & 4 & 1 & 1 & 1 & 1 & 0 & 1 & 1 \\
\hline 9 & 4 & $2^{a}$ & 1 & 1 & 1 & 1 & 0 & 1 & $2^{b}$ \\
\hline 10 & 1 & 1 & 1 & 1 & 1 & 1 & 0 & 1 & 1 \\
\hline 11 & 2 & $1^{a}$ & 2 & $\mathbf{1}^{a}$ & 2 & $1^{a}$ & 1 & 1 & 1 \\
\hline 12 & 4 & 4 & 3 & $1^{a}$ & 6 & $1^{a}$ & 5 & 3 & 3 \\
\hline 14 & 4 & $2^{a}$ & 1 & 1 & 1 & 1 & 0 & 1 & 1 \\
\hline 15 & 4 & $2^{a}$ & 2 & $\mathbf{3}^{b}$ & 2 & $\mathbf{8}^{b}$ & -6 & 1 & $2^{b}$ \\
\hline 16 & 2 & $\mathbf{1}^{a}$ & 2 & $\mathbf{1}^{a}$ & 2 & $1^{a}$ & 1 & 1 & 1 \\
\hline 17 & 1 & 1 & 3 & $2^{a}$ & 7 & $5^{a}$ & 2 & 4 & 4 \\
\hline 18 & 4 & 4 & 2 & $\mathbf{1}^{a}$ & 4 & $1^{a}$ & 3 & 2 & $3^{b}$ \\
\hline 19 & 4 & 4 & 3 & $1^{a}$ & 8 & $1^{a}$ & 7 & 1 & $2^{b}$ \\
\hline 20 & 1 & $\mathbf{2}^{b}$ & 3 & $2^{a}$ & 8 & $2^{a}$ & 6 & 3 & 3 \\
\hline 21 & 4 & $\mathbf{2}^{a}$ & 3 & $\mathbf{2}^{a}$ & 8 & $4^{a}$ & 4 & 2 & 2 \\
\hline 22 & 4 & $2^{a}$ & 1 & 1 & 1 & 1 & 0 & 1 & 1 \\
\hline 23 & 4 & $2^{a}$ & 1 & 1 & 1 & 1 & 0 & 1 & 1 \\
\hline 24 & 2 & 2 & 2 & $\mathbf{1}^{a}$ & 2 & $\mathbf{1}^{a}$ & 1 & 1 & 1 \\
\hline 26 & 4 & $2^{a}$ & 1 & 1 & 1 & 1 & 0 & 2 & 2 \\
\hline 29 & 4 & $1^{a}$ & 3 & $1^{a}$ & 8 & $\mathbf{1}^{a}$ & 7 & 1 & 1 \\
\hline
\end{tabular}

$C A R B$ carbonated thin liquids, NOCARB noncarbonated thin liquids

See Table 2 for definitions of outcome measures

${ }^{a}$ Indicates patient swallows improved with CARB

$b_{\text {Indicates patient swallows worsened with CARB }}$

Bold text indicates change 